\title{
Criminologie
}

\section{La résistance à la pénalité : un impératif féministe}

\section{Karlene Faith}

Volume 35, numéro 2, automne 2002

Femmes et enfermement au Canada : une décennie de réformes

URI : https://id.erudit.org/iderudit/008293ar

DOI : https://doi.org/10.7202/008293ar

Aller au sommaire du numéro

Éditeur(s)

Les Presses de l'Université de Montréal

ISSN

0316-0041 (imprimé)

1492-1367 (numérique)

Découvrir la revue

Citer cet article

Faith, K. (2002). La résistance à la pénalité : un impératif féministe.

Criminologie, 35(2), 115-134. https://doi.org/10.7202/008293ar

\section{Résumé de l'article}

À l'ère de la croissance galopante des prisons sous forme de complexe industriel en Amérique du Nord, les tensions idéologiques vacillent entre châtiment et mouvements en faveur d'une justice réparatrice ou transformatrice. Entre les désinstitutionnalistes, qui cherchent à réduire le nombre de personnes en prison, et les abolitionnistes pénaux, qui visent une transformation sociale, on retrouve nombre de féministes canadiennes.

Les femmes au Canada sont désillusionnées suite à l'échec du gouvernement fédéral à mettre en pratique la vision de La création de choix, un rapport d'un groupe de travail sur l'emprisonnement des femmes, datant de 1990. Le gouvernement n'a pas non plus tenu compte des importantes recommandations de la juge Louise Arbour, dont l'enquête de 1996 sur les inconduites du personnel correctionnel met en lumière le non-respect des lois dans les prisons. En 2002, encore plus de femmes se retrouvent dans les prisons à sécurité moyenne ou maximale au Canada pour des crimes relativement mineurs. Les années 1990 nous ont fourni une bonne leçon sur la futilité de la réforme des prisons, tout spécialement au nom du féminisme. Dans l'avenir, les féministes devront impérativement contester les fondements de la pénalité imposée par l'État plutôt que de tenter de réformer une institution désuète.
Ce document est protégé par la loi sur le droit d'auteur. L'utilisation des services d'Érudit (y compris la reproduction) est assujettie à sa politique d'utilisation que vous pouvez consulter en ligne.

https://apropos.erudit.org/fr/usagers/politique-dutilisation/ 


\title{
La résistance à la pénalité : un impératif féministe ${ }^{1}$
}

\author{
Karlene Faith \\ Professeure \\ École de Criminologie \\ Université Simon Fraser \\ faith@sfu.ca
}

RÉSUMÉ • À l'ère de la croissance galopante des prisons sous forme de complexe industriel en Amérique du Nord, les tensions idéologiques vacillent entre châtiment et mouvements en faveur d'une justice réparatrice ou transformatrice. Entre les désinstitutionnalistes, qui cherchent à réduire le nombre de personnes en prison, et les abolitionnistes pénaux, qui visent une transformation sociale, on retrouve nombre de féministes canadiennes.

Les femmes au Canada sont désillusionnées suite à l'échec du gouvernement fédéral à mettre en pratique la vision de La création de choix, un rapport d'un groupe de travail sur l'emprisonnement des femmes, datant de 1990. Le gouvernement n'a pas non plus tenu compte des importantes recommandations de la juge Louise Arbour, dont l'enquête de 1996 sur les inconduites du personnel correctionnel met en lumière le non-respect des lois dans les prisons. En 2002, encore plus de femmes se retrouvent dans les prisons à sécurité moyenne ou maximale au Canada pour des crimes relativement mineurs. Les années 1990 nous ont fourni une bonne leçon sur la futilité de la réforme des prisons, tout spécialement au nom du féminisme. Dans l'avenir, les féministes devront impérativement contester les fondements de la pénalité imposée par l'État plutôt que de tenter de réformer une institution désuète.

ABSTRACT - In an era of runaway growth of the North American prison industrial complex, ideological tensions lurk between penality and movements toward restorative or transformative justice. Decarcerationists, who seek to reduce the numbers of people in prison, and penal abolitionists, who seek social transformation, include many Canadian feminists.

Women in Canada are disillusioned by the failure of the federal government to implement the vision of Creating Choices, a 1990 task force report on women's imprisonment. Nor did it heed significant recommendations by the Honourable Louise Arbour,

1. Texte traduit par André Bourbonnière. 
whose 1996 inquiry into wrongdoing by correctional staff documents the lawlessness of prisons. In 2002, more women in Canada are being incarcerated in medium and maximum security prisons for relatively minor crimes. An important lesson from the $1990 \mathrm{~s}$ is the futility of prison reform, especially in the name of feminism. A key feminist imperative for the future is to challenge state penality at its core rather than attempt to reform an outdated institution.

\section{Introduction}

Deux thèmes, à la fois distincts et reliés, sont à la base du présent mémoire. Premièrement, je passe en revue l'historique récent des prisons fédérales pour femmes au Canada, et les tentatives manquées pour réformer une institution fondamentalement punitive. Deuxièmement, j'examine des défis à la présomption pratiquement universelle qui veut que la punition soit la réponse appropriée à un comportement illégal.

En Occident, les idéologies pénales et les techniques punitives de l'État se sont transformées avec le temps; toutefois, l'accent a été mis invariablement sur l'imposition de la souffrance, en recourant au stigmate, à l'humiliation, à la privation, à l'incarcération, à la torture et, jusqu'à tout récemment, à différentes méthodes d'exécution.

On n'attache plus les prostituées au pilori de la grand-place pour leur lancer des légumes pourris, des pierres et des insultes. Les épouses qui harcèlent leurs maris n'ont plus à porter une bride de mégère. On n'attache plus les femmes soupçonnées d'actes contre nature à un tabouret pour les plonger en eau profonde. On ne brûle plus les femmes sur le bûcher pour sorcellerie, on ne les envoie plus à la guillotine pour avoir volé ou tué leur mari, on ne les plonge plus dans l'huile bouillante pour avoir manqué de respect au roi. À l'exception des États-Unis (où l'on exécute encore les criminels par pendaison, chaise électrique, peloton d'exécution, chambre à gaz ou injection dans 38 des 50 États), toutes les nations occidentales ont abandonné la peine capitale. Au lieu de s'adonner à un barbarisme flagrant, on enferme maintenant les gens.

Depuis leur prolifération au XIX ${ }^{\mathrm{e}}$ siècle, les prisons ont joué bien des rôles : alternative à la peine de mort; moyen d'entreposer les indésirables, particulièrement les minorités discriminées; extension de la bureaucratie étatique et des systèmes de classification; travaux forcés; laboratoire pour les chercheurs en sciences sociales et les «psy» professionnels; riche filon d'anges déchus pour évangélistes et réformateurs de la morale; et, fondamentalement, punition. Comme ce fut le cas dans les châtiments d'antan, les prisons modernes stigmatisent, humilient, pri- 
vent, torturent insidieusement par des techniques telles que le garde-àvue 24 heures sur 24 en cellule d'isolement.

Les prisons réussissent à châtier, mais elles n'arrivent pas à dissuader ni à empêcher le crime, les contrevenants condamnés à la prison ne ressortant généralement ni corrigés ni réhabilités. Les prisons protègent parfois le public, du moins temporairement, de ceux qui constituent un danger pour autrui. Mais la majorité des gens en prison ne sont pas dangereux; ceci est particulièrement vrai des femmes incarcérées. Les prisons constituent une importante industrie en croissance en Amérique du Nord, non parce qu'elles sont efficaces contre les infractions à la loi ou qu'elles font évoluer la justice, mais parce qu'elles représentent l'appareil pénal établi par l'État, qu'elles emploient une forte main-d'œuvre, et profitent aux entreprises de construction, de quincaillerie et de services.

L'histoire des prisons est une histoire de tentatives de réformes infructueuses. Le fait que tant de gens possèdent des investissements dans l'industrie de la punition est une des causes principales de cet insuccès. Gayle Horii, qui a observé les prisons sur place pendant sept ans, nous livre ces commentaires :

L'axiome de la priorité de la prison «pour le bon ordre de l'institution» donne une mauvaise impression de la véritable mission (le discours privé) des autorités «correctionnelles» - croissance de leur «industrie», sécurité d'emploi et sûreté, dissimulation. Les gens employés par ou sous-contrat pour le compte du SCC [Service correctionnel du Canada] doivent déclarer que «la non divulgation des torts» appelée «confidentialité», est une condition acceptable d'embauche. Cette situation fait que certains des crimes les plus odieux continuent à être commis, au nom de l'État (Horii, 2000 : 112; notre traduction).

Dans le présent texte, je traite des prisons pour femmes au Canada. Mais il est possible de généraliser ces conclusions. La majorité des prisonnières sont de facto impuissantes, affligées d'un passé de victimisation. Il n'est pas logique de s'attendre à ce que les personnes emprisonnées expriment leur gratitude à ceux qui les enferment, les privent d'agréments physiques, les mettent en isolement, leur refusent de faire un téléphone ou d'obtenir une absence temporaire pour assister à des funérailles dans la famille, ou les avilissent ou les irritent de quelque façon. Il est également déraisonnable de s'attendre à ce que les gens en position de force sur des êtres humains démontrent du respect envers des captives qui ont déjà été condamnées et étiquetées comme criminelles par l'État, spécialement si elles font preuve de «mauvaise attitude». C'est là une situation intenable, responsable du nombre élevé de suicides et d'autres 
morts parmi les prisonnières et les femmes remises en liberté depuis peu, ainsi que de la forte proportion d'alcoolisme et d'autres problèmes personnels chez les gardiens de prison (Faith, 1993).

La discussion suivante sur les prisons pour femmes au Canada tient compte de la rudesse et de l'insensibilité de la vie en prison. Étant donné que l'emprisonnement est néfaste pour l'esprit humain et le caractère d'une nation, je porterai mon attention, dans la deuxième partie, sur les approches non pénales face au crime. Depuis des décennies, autant les désinstitutionnalistes, qui cherchent à réduire le nombre de personnes incarcérées, que les abolitionnistes, qui fermeraient toutes les prisons sauf pour les individus véritablement dangereux, préconisent des solutions au sein de la communauté.

\section{Les femmes : une énigme correctionnelle}

Entre 1938 et 1980, 13 comités, commissions ou groupes de travail nommés par le gouvernement se sont penchés sur la question de savoir si la prison des femmes de Kingston, en Ontario (PdF), répondait aux normes correctionnelles fondamentales. Chaque fois, l'institution a échoué, et les examinateurs ont chaque fois recommandé la fermeture de la tristement célèbre PdF, qui, depuis plus de 50 ans, était la seule prison canadienne occupée exclusivement par des femmes condamnées à des peines fédérales. Depuis des décennies, on insistait, en vain jusque dans les années 1990, auprès du SCC pour que la PdF soit remplacée par de petites résidences régionales, à sécurité minimale, où les femmes seraient plus près de leurs familles. En 1989, le Solliciteur général nommait un autre groupe de travail en vue d'effectuer des recommandations concernant les «mesures de correction pour les femmes» au Canada. Encouragé par l'Association canadienne des sociétés Elizabeth Fry (ACSEF) et des organisations de femmes autochtones, ce groupe de travail sur les femmes condamnées à des peines fédérales différait de ses prédécesseurs; le groupe de quarante-cinq membres était composé majoritairement de femmes, représentant les Premières Nations, de francophones, de deux ex-prisonnières, de féministes, de groupes militant à l'échelle nationale ou régionale, et de professionnelles de la justice criminelle. Fait très important, le groupe de travail rencontra la majorité des femmes alors incarcérées ou en libération conditionnelle.

Intitulé La création de choix et publié le 19 avril 1990, le rapport comportait entre autres les thèmes suivants : 1) une forte majorité des pri- 
sonnières, particulièrement les femmes autochtones, avaient subi des abus sexuels et physiques, la plupart dès l'enfance; ces femmes avaient besoin de trouver des moyens de guérir; 2) les femmes incarcérées ont besoin d'avoir accès à des ressources au sein de la communauté telles que formation professionnelle, counseling, formation scolaire, services légaux, aide pour l'éducation des enfants, programmes de guérison pour les abus de drogue ou d'alcool,etc.; 3) les mères avaient besoin d'être près de leurs enfants; 4) les femmes autochtones, de même que les femmes noires et les autres minorités, ont des besoins différents et ont droit à leur héritage culturel, le personnel devant respecter et comprendre leur diversité.

Le Service correctionnel du Canada (SCC) accepta de fermer en 1994 l'archaïque prison des femmes, ce qui n'advint qu'en mai 2000, après plusieurs délais. Entre-temps, soit entre 1995 et 1997, cinq nouvelles institutions régionales pour femmes ont vu le jour à (ou près de) Edmonton, en Alberta; Kitchener,en Ontario; Joliette, au Québec; Truro, en Nouvelle-Écosse; et Maple Creek, en Saskatchewan. La plupart sont isolées et certaines sont loin des arrêts d'autobus municipaux, ce qui limite les visiteurs. Bien qu'à l'origine ces établissements étaient des prisons à sécurité minimale, construites dans le style de maisons de ville, après plusieurs tentatives d'évasion et un meurtre (ou suicide assisté), on ajouta bientôt des clôtures de sécurité maximale, des murs, des verrous électroniques, des lasers, des caméras, des détecteurs de métal, une surveillance humaine omniprésente et des politiques de punition conventionnelle sous forme d'isolement. La paranoïa ressentie dans ces prisons pastel se reflète dans la quincaillerie de sécurité superflue, image de danger qui vient renforcer l'idéologie de punition. L'appui initial apporté par nombre de féministes et d'autres personnes en faveur «d'installations» de remplacement indiquait leur complicité involontaire avec le retour de la légitimation des prisons.

À la fin des années 1990, la plupart des occupantes des prisons pour femmes avaient été transférées dans l'une de ces nouvelles prisons régionales, alors que les autres prisonnières étaient conduites dans des unités de sécurité maximale au sein de prisons pour hommes. En ColombieBritannique, les femmes condamnées à des peines fédérales purgeaient leur sentence au Centre correctionnel pour femmes de Burnaby, géré par la province pour les incarcérations de ressort fédéral comme provincial. Nancy Wrenshall, directrice régionale du Service correctionnel pour femmes de la Colombie-Britannique, adopte la position que la punition réside dans le fait d'être enfermée, et qu'il n'appartient pas à la prison 
d'infliger d'autres châtiments. Malgré cela, tout comme dans les prisons régionales, le personnel du BCCW a recours aux atomiseurs de poivre quand les femmes se montrent peu coopératives.

La révélation au grand jour d'un drame de brutalité contre les prisonnières de la PdF accéléra la construction de cinq nouvelles prisons. En avril 1994, après deux jours de protestations de la part des prisonnières et de gestes d'hostilité provenant des gardiens, une équipe d'intervention (provenant du pénitencier pour hommes situé sur la même rue) fit irruption dans l'unité de ségrégation de la PdF où huit prisonnières, dont six autochtones, étaient déjà couchées. Une par une, ces femmes furent soumises à des fouilles à nu, afin, de toute évidence, de trouver de objets de contrebande. Une équipe d'hommes (et une femme) masqués, en uniformes de Darth Vader, ont tenu sur le plancher de ciment des femmes petites et non violentes, déchirant leurs vêtements jusqu'à ce qu'elles soient nues. Certaines des femmes criaient : leurs attaquants restaient silencieux. L'une des femmes a supplié qu'on lui donne ses lunettes; en guise de réponse, les lunettes furent écrasées sous une botte. Menottes aux poings, enchaînées aux chevilles et à la taille, à peine couvertes de robes de papier, elles furent projetées, face première, contre le mur de ciment pendant que l'équipe de fouille faisait claquer leurs bâtons sur le mur près des oreilles de leurs victimes. Tour à tour, les femmes furent emmenées dans une autre pièce et soumises à une fouille vaginale. On ne trouva aucune contrebande. Les femmes furent mises en isolement, certaines jusqu'à neuf mois, caméras et lumière constamment sur elles, sous garde surveillée 24 heures par jour. Pendant un certain temps, elles furent même privées de tout bien matériel tels que vêtements, matelas, couvertures, produits d'hygiène, matériel pour écrire et téléphones.

Comme c'est le cas pour toutes les interventions de l'équipe d'urgence, ces fouilles à nu furent filmées sur vidéo et la bobine clandestinement envoyée à la chaîne de télévision CBC. Suivant la menace de procès où le SCC tenta d'empêcher une projection publique, la vidéo passa en ondes en janvier 1995, dans le cadre de l'émission The Fifth Estate. Le public se montra outragé que des femmes, fussent-elles incarcérées, soient brutalisées par des employés du gouvernement dans l'exercice de leurs fonctions. Des ex-prisonnières vinrent témoigner que de tels événements n'étaient pas inhabituels.

Le Solliciteur général nomma un comité d'enquête sous la présidence de la juge Louise Arbour, qui allait plus tard agir comme juge en chef au tribunal des crimes de guerre à La Haie, et qui siège aujourd'hui à la 
Cour Suprême du Canada. Celle-ci condamna sans équivoque les événements survenus en avril 1994, en plus de critiquer la façon généralement illégale et mensongère dont était gérée la prison. Son rapport (Arbour, 1996), préconisant un examen judiciaire minutieux, établit une accusation vigoureuse face à la responsabilité du SCC, mais, tout comme ce fut le cas pour le groupe de travail de 1990, quelques recommandations seulement furent prises en compte. Dans toutes les prisons régionales, des gardiens montent la garde, les femmes à problème sont placées en isolement dans des cellules vidées de leur contenu pour des raisons disciplinaires, administratives, médicales ou de protection; les femmes incontrôlables sont aspergées de poivre; et les prisons sont gouvernées de façon hiérarchique sur le principe de la punition face aux infractions.

\section{La loge de guérison : lumière au bout du tunnel ?}

Parmi les recommandations que l'on a ignorées, la juge Arbour conseillait au SCC d'adapter toutes les nouvelles prisons régionales à la philosophie de guérison, telle que pratiquée par la loge de guérison, alors nouvelle, sur le territoire de Nekaneet, près de Maple Creek, en Saskatchewan. Contrairement aux autres prisons régionales, cette institution a été conçue d'après les recommandations d'un groupe de travail prônant l'établissement, pour les femmes autochtones, d'une institution adaptée à leur culture et basée sur le processus de guérison propre à celle-ci. Environ $20 \%$ des femmes condamnées à des peines fédérales au Canada sont d'origine autochtone, bien qu'elles ne forment que $4 \%$ de la population canadienne. Entre la fin des années 1970 et les années 1990, une douzaine de femmes se sont suicidées à la PdF; huit étaient autochtones (Faith et al., 1990; Sugar et Fox, 1989-1990). Si ces femmes subissent de longues sentences, ce n'est pas en raison de la sévérité de leur crime, mais parce qu'elles ne peuvent s'adapter aux prisons de leurs colonisateurs.

Diverses communautés des Premières Nations, particulièrement dans le Nord, ont obtenu le droit d'administrer leur propre justice, souvent dans des cérémonies du cercle de guérison auxquelles assistent des juges itinérants ainsi que des représentants de la communauté, des Anciens, et toutes les parties directement touchées par le délit. Le principe de la loge de guérison, avec plusieurs membres des Premières Nations dans son personnel et la participation des Anciens de Nekaneet, reposent sur les traditions culturelles qui visent l'harmonie de la communauté plutôt que le châtiment de confrontation. L'administratrice en chef est une Kikawinaw 
(«notre mère» en Cree), plutôt qu'une directrice, pour faire comprendre qu'il s'agit d'un refuge. Aucun membre du personnel n'a de formation sur l'utilisation d'armes, ce qui serait incompatible avec les buts, la structure, le concept et la philosophie de la loge. Les femmes qui purgent ou ont purgé une sentence à cet endroit peuvent raconter comment elles y arrivent à guérir des effets négatifs du bouleversement culturel, un phénomène répandu parmi les autochtones du Canada.

La loge constitue un modèle visionnaire, conçu par Patricia Monture et développé avec l'appui d'autres femmes des Premières Nations sur le groupe de travail. Ce groupe avait recommandé l'admission des femmes démontrant le plus vif besoin d'un environnement de guérison, quelle que soit leur classification. Toutefois, en six ans d'existence, on n'y a admis que les femmes déjà classifiées à sécurité minimale, c'est-à-dire considérées comme détenues dociles.

Planifiée comme une rupture franche avec la philosophie correctionnelle (Faith, 1995), la loge agit comme un havre d'espoir. Cependant, son existence ne signifie pas encore l'abandon généralisé du système punitif. La loge n'a été prévue que pour accueillir trente femmes; en 2002, on n'y dénombrait que vingt résidentes. En théorie, les femmes peuvent avoir leurs enfants avec elles; mais lorsque Patricia Monture et moi avons visité l'endroit, en mai 2002, il n'y avait aucun enfant. Bien que l'existence même de la loge soit un compromis par rapport à la tradition pénale, le SCC agit de toute évidence avec prudence (Monture-Angus, 2000). Par exemple, le SCC s'enorgueillit des 160 acres de boisé octroyés par le Nekaneet au profit de la loge de guérison, mais les femmes restent confinées à l'espace autour des bâtiments. Une barrière électronique empêche toute personne de pénétrer sur les lieux sans contrôle d'identité préalable. Des gardiens spéciaux émettent des règlements sans importance à gauche et à droite, de sorte que l'accent sur la sécurité n'a pas souffert de compromis dans cette entreprise correctionnelle par ailleurs innovatrice. Mais, en même temps, le succès de la loge, qui insiste sur le rétablissement culturel, la guidance spirituelle selon la tradition et la responsabilité de l'individu face à sa communauté, établit une faille dans le système correctionnel basé sur la punition.

D'un point de vue personnel, notre visite à la loge reste mémorable. Il tombait une petite neige de printemps et le feu brûlait dans un poêle à tuyau au centre de la pièce, dans cette haute structure de cèdre, en forme de tipi; nous étions installées confortablement sur des bancs en cercle pendant que six femmes chantaient des chants sacrés en battant du 
tambour. D'autres femmes nous ont raconté leur histoire. Nous nous sommes jointes aux femmes pour la cérémonie de purification où l'on brûle du cèdre et de la sauge dans un coquillage. Aucun membre du personnel n'était présent. Avec nos yeux et nos oreilles, nous avons pu comprendre pourquoi la loge de guérison constitue une approche beaucoup plus significative pour prévenir la récidive que la prison conventionnelle. Pour couronner cette ambiance spirituelle, sur le chemin du retour vers Maple Creek, dans la nuit silencieuse sur une petite route de campagne enneigée, nous avons aperçu une quinzaine de cerfs sortant des bois. J'ai visité plus d'une prison dans ma vie, mais c'était la première fois que je sortais d'une prison en me sentant apaisée.

\section{Situation actuelle des femmes canadiennes en prison}

Alors que le groupe de travail cherchait à réduire les éléments les plus sévères de la punition, on délaissa la question même de l'incarcération. En fermant la PdF, on disposait alors de beaucoup plus de cellules libres, ce qui eut pour conséquence d'augmenter le nombre de femmes incarcérées (en 2002, 370 femmes emprisonnées servent des sentences fédérales). Les cinq nouvelles prisons permettent de disposer de plus d'espace pour incarcérer plus de femmes pendant des périodes plus longues au sein d'institutions infantilisantes (Kendall, 1994; Hannah-Moffat, 2001). Ce qui est plus grave encore, on a aussi incarcéré des femmes dans des institutions pour hommes, où elles sont droguées et mises en isolation dans des ailes psychiatriques, comme le pénitencier et le Regional Psychiatric Centre en Saskatchewan, la prison de Sainte-Anne-des-Plaines au Québec, et la Springhill Institution en Nouvelle-Écosse.

Pendant ce temps, on construit des pavillons cellulaires à sécurité maximale, à l'écart sur le terrain de quatre des nouvelles prisons pour femmes (exception faite de la loge de guérison). L'intention est de séparer les prisonnières considérées, souvent incorrectement, comme souffrant de troubles psychiques (ou dont les troubles découlent des conditions de l'incarcération), ou classifiées comme présentant un risque d'évasion ou une menace au bon fonctionnement de l'institution. Chacune des prisons régionales dispose déjà de cellules d'isolement appelées «unités d'encadrement renforcé» auxquelles viendront se greffer ces nouvelles unités à sécurité maximale. En 2001, 18 femmes seulement étaient classifiées à sécurité maximale, en majorité des autochtones, la plupart d'entre 
elles ne représentant aucun danger pour les autres. Le fait d'accroître le nombre d'unités d'isolement n'est évidemment pas une question de sécurité; il semblerait plutôt que le SCC reste sur ses positions d'approche punitive et discriminatoire quant aux mesures correctionnelles (HannahMoffat et Shaw, 2000; Hannah-Moffat, 2001; Shaw et Hannah-Moffat, dans le présent numéro).

Si les prisons parviennent rarement à la réhabilitation, c'est que guérison et punition sont des approches antithétiques, fonctionnant généralement à l'encontre l'une de l'autre, irréconciliablement. Voici exactement ce que déclarait un médecin consulté par le comité de planification d'une prison provinciale de la Nouvelle-Écosse en matière de thérapie :

À la lumière des connaissances actuelles sur la psychologie des femmes, il est évident que la prison ne peut constituer un endroit de guérison pour les femmes. [...] C'est une chose que de créer en prison un environnement qui tente de minimiser la détresse émotionnelle découlant de l'incarcération, et tout à fait une autre que de fournir un endroit où les femmes peuvent entreprendre leur guérison (SGSCPIW, 1992 : Appendice pp. 2 et 5; notre traduction).

La nouvelle chaîne de prisons pour femmes est conventionnellement pénale quant à sa philosophie et à ses pratiques, si ce n'est dans son architecture. Telle est la raison, et le résultat, de la décision des fonctionnaires du SCC de ne pas intégrer les membres du groupe de travail dans le processus d'implantation. En effet, le mandat original du groupe de travail se situait à l'intérieur du modèle correctionnel. On a particulièrement exclu toute représentation de l'Association canadienne des sociétés Elizabeth Fry, et des membres des Premières Nations participant au groupe de travail, bien que leur initiative soit à l'origine même de cette étude (Shaw, 1993). On écarta également la vision féministe. Les fonctionnaires du Service correctionnel du Canada avaient l'occasion de sortir les femmes de l'imbroglio de l'entreprise correctionnelle; au lieu de cela, ils ont réinséré des idéologies carcérales en reproduisant des politiques pénales anachroniques.

\section{Le commencement de la fin des prisons}

À l'échelle mondiale, simultanément à la croissance de l'industrie des prisons, et en réponse à cette croissance, le mouvement international en faveur de l'abolition des prisons (ou, plus radicalement, l'abolition pénale) démontre une recrudescence de la résistance à l'emprisonnement comme solution à la criminalité (voir Mathiesen, 1986; 1990; de Haan, 1990; 
Quinney et Pepinsky, 1990 ; Christie, 1993 ; MacLean et Pepinsky, 1993). Le but ne réside pas tout entier dans la désinstitutionnalisation, c'est-à-dire dans la réduction du nombre de personnes en prison, mais dans le passage d'une société punitive vers une société visant à établir des communautés.

En 1971, le American Friends Service Committee (Quakers) publiait une analyse critique de la justice criminelle, proposant des réformes radicales tel que le «recours en dernier lieu à la police, aux cours et aux prisons pour venir à bout de problèmes qui ne peuvent être traités de façon plus humaine» (AFSC, 1971 :171). Cette analyse concluait par une perspective presciente de la désinstitutionnalisation :

Quand des pressions pour des réformes mènent à des exigences de remédier à la «surpopulation» en ajoutant cellules et lits, le résultat est inévitable : le filet coercitif du système judiciaire va s'abattre sur un plus grand nombre de personnes, les prenant au piège pour une plus longue durée. Si les prisons sont surpeuplées, trouvons des moyens de réduire la masse des lois criminelles et des mesures coercitives qui envoient tant de personnes en prison (AFSC, $1971: 172-73$ ).

Durant les années 1970, le document de l'AFSC était une lecture essentielle dans le mouvement pour les droits des prisonniers; il constitue toujours un guide indispensable du militantisme. Voici certaines de leurs recommandations : 1) appliquer les lois de la même manière, pour les riches et pour les pauvres; 2) séparer le traitement de la punition; 3 ) reconnaitre aux prisonniers leurs droits en tant que personnes; 4) faire participer la communauté, prisonniers inclus, à la reconstruction du système de justice. Plutôt que de perpétuer la tradition des réformes libérales des Quakers, ils mettent l'accent sur la futilité de la réforme (AFSC, $1971: 146)$.

Depuis plus d'un siècle, les réformateurs tentent de sauver les femmes par la religion, l'alphabétisation, le travail et, à partir des années 1950, la thérapie psychologique, étouffant les appels à l'équité économique, sociale et politique et à la réduction de l'emprisonnement. S'appuyant sur une étude menée à la PdF, Kendall observe comment on ne peut considérer une activité comme thérapeutique que si elle offre aux participantes «un espace où elles peuvent être elles-mêmes (un répit de ce sentiment de constante surveillance), avoir le contrôle de leur propre vie et/ou la possibilité de valoriser les autres et d'être aussi valorisées». Ces éléments «sont en opposition directe avec la nature même des prisons» (Kendall, 1994 : 11; notre traduction; voir aussi Kendall, 2000, et Balfour, 2000). 
En 1962, le réformateur et planificateur de prisons Joseph Eaton insistait sur «le contrôle absolu» qu'administrateurs et gardiens exercent sur la vie des prisonniers, même lorsqu'ils sont ouvertement engagés dans un travail scientifique de réhabilitation. Eaton illustre comment les discours qui en surface militent pour l'âme, l'esprit et le corps du prisonnier, sont en fait mutuellement complémentaires, renforçant la fonction de captivité et de punition des prisons. Les contraintes se resserrent au fur et à mesure que les professionnels rivalisent pour obtenir le pouvoir sur le prisonnier.

On peut considérer le monde des prisons et ses objectifs, ainsi que la resocialisation des détenus, comme une arène où les représentants des diverses professions d'aide interagissent dans un effort pour s'occuper d'une tâche très complexe : refaire les déviants, et s'il est peu probable qu'on arrive à les refaire, les contrôler de façon efficace. [...] Psychiatres, psychologues, travailleurs sociaux, éducateurs, professeurs de formation professionnelle et autres spécialistes [...] sont à la recherche de moyens plus efficaces de refaire les prisonniers afin de répondre aux attentes de la société (Eaton, $1962: 182$; notre traduction).

Ignorant le contexte social, ces «professions d'aide» individualisent le crime, considèrent le délinquant comme un malade, et concourent à la réglementation sociale, tout en soutenant le système pénal.

Dans son étude des nations avec un taux de criminalité faible, Freda Adler (1983) s'est aperçue que les taux les plus faibles correspondaient à un niveau élevé d'engagement interpersonnel et de responsabilité directe face à ses pairs et à sa culture, ce qui constitue des caractéristiques clés de la communauté. La communauté s'occupe des besoins individuels de ses membres et, dans l'esprit du contrat social, chaque membre contribue à la communauté et se conforme aux lois sociétales et aux pressions sociales informelles pour obtenir sécurité et protection. Parler d'abolir les prisons, c'est imaginer remplacer l'isolationnisme et l'individualisme de l'organisation capitaliste de la société par la collectivité et l'effort mutuel, à l'intérieur des cultures et entre elles.

Les désinstitutionnalistes préconisent de trouver des solutions aux problèmes au sein de la communauté, avant que ne soient commis les crimes et pour éviter la récidive. Toutefois, tout le monde ne fait pas partie d'une communauté. Comme le font remarquer Chan et Ericson (1981:6), les prisons sont peuplées de gens dont les communautés ont été fragmentées. C'est avoir la vue courte que de s'attendre à ce qu'une communauté résolve un problème de criminalité lorsqu'elle est elle-même la source du crime. Avec l'urbanisation et la migration en masse, toutes les notions traditionnelles de communauté subissent une érosion. Le processus de réduction à 
la fois des impulsions de l'individu vers l'illégalité et du pouvoir de l'État de punir de façon sélective, ne se fera pas sans une reconfiguration et un renforcement de diverses communautés, au niveau régional ou mondial.

Le modèle réparateur, qui s'inspire en partie des traditions aborigènes, prévoit que la ou les victime(s), le délinquant et la communauté travailleront ensemble pour assurer la responsabilisation, la restitution, la guérison et la réintégration. Ce n'est qu'en faisant régner un climat général de dignité qu'on arrivera à éviter les cycles de transgression et de vengeance.

Le modèle Quaker de justice réparatrice, tel que décrit ci-après, partage certaines caractéristiques avec les Premières Nations, et établit les normes suivantes :

1. Le délinquant est responsable du mal causé, mais il est incité à en prendre conscience et à réparer ce qu'il peut.

2. La victime reconnaît le mal qu'on lui a causé et participe au processus de réparation.

3. La communauté joue un rôle dans l'établissement de l'harmonie et de l'intégration (Morris, 1992).

Le modèle transformateur s'écarte de la justice réparatrice en faisant valoir que les iniquités sociales basées sur la classe et la race entraînent des processus de criminalisation et d'incarcération sélectives. Le but est de transformer les relations sociopolitiques qui produisent autant les comportements illégaux que les réponses discriminatoires. Alors que la justice réparatrice est une tactique de désinstitutionnalisation utile et à court terme pour désinstitutionnaliser la punition, la justice transformatrice est une stratégie à long terme visant l'abolition des prisons.

\section{Approches féministes}

Généralement, les féministes activistes appuient les abolitionnistes en ce qui a trait aux femmes, tout comme nombre de criminologues féministes. Ainsi, Dobash, Dobash et Gutteridge en arrivent à la conclusion que «l'emprisonnement est en soi une réponse à la fois inappropriée et nocive face à la criminalité des femmes» (1986:214; notre traduction). Carlen ne prévoit pas que les prisons, symboles de la «culture masculine», puissent jamais convenir aux femmes. Elle préconise les foyers pour femmes pour favoriser une forme d'autoautorité parmi des femmes qui comprennent leurs situations réciproques. Étant donné que peu de femmes constituent une 
menace, Carlen soutient qu' «il serait initialement plus facile de convaincre la population de tenter d'abolir les prisons pour femmes que les prisons pour hommes» (Carlen, 1990 : 121 ; notre traduction). Brian MacLean et Hal Pepinsky font remarquer que «la pensée féministe non seulement influence notre position abolitionniste, mais elle y est essentielle» (1993 : 10 ; notre traduction). Confronter les prisons revient à confronter un pouvoir pénal patriarcal, militariste, hiérarchique.

Cette hésitation des féministes face à l'abolition des prisons pour hommes révèle l'énigme analytique et légale de la neutralité des genres (similitude) face à la spécificité des genres (différence). Bien des féministes qui prêchent en faveur de l'abolition des prisons pour femmes restent silencieuses face à l'incarcération des hommes, parce que plusieurs d'entre eux représentent une menace à la sécurité des femmes et des enfants. Toutefois, Knopp présente une perspective et une pratique abolitionnistes féministes radicales, en faisant valoir des cas spécifiques de viols et d'abus sexuels (Knopp et al., 1976; 1993). Elle met en évidence ces crimes qui ont généralement entraîné une réaction d'endurcissement chez les féministes et chez les femmes, pour démontrer que même avec les crimes de violence chez les hommes, les programmes au sein de la communauté sont plus efficaces que la prison (Knopp, 1993 : 55-56). Selon la stratégie d'usure de Knopp (contrôle de quelques-uns), elle placerait les délinquants sexuels dans des résidences de rééducation «au premier signe d'agression sexuelle» (Knopp, 1993 : 59). Knopp et d'autres activistes travaillant avec des délinquants sexuels sont persuadés que les réponses «les moins restrictives» et «les plus humaines», dans le cadre d'un environnement de rééducation (féministe) sont plus efficaces que la punition catégorique, bien que la contrainte soit une caractéristique fondamentale de la rééducation forcée.

D’une façon générale, Greenberg et Stender soutiennent que «la prison opprime en effet non seulement ceux qui se retrouvent sous les verrous, elle atteint chacun de nous» (1972: 886; notre traduction). Auteure de la première étude d'envergure sur les prisons pour femmes aux ÉtatsUnis, Kathryn Burkhart prétend également que les prisons sont nocives pour la société, et sont auto-destructrices : «Nous utilisons de l'énergie et des ressources précieuses pour nous infliger du mal à nous-mêmes » (Burkhart, 1973 : 427; notre traduction). Elle cite un juge de l'Ohio qui, en 1870, prédisait que les «prisons seraient abolies quand les femmes auraient acquis le droit de vote, occuperaient des charges administratives et siégeraient en qualité de juges et de législateurs» (Ibid., 425). 
Durant les années 1990, l'intérêt pour des modèles de justice négociée gagne de la popularité au Canada. Avec des programmes approuvés par les cours et financés par l'État, diverses communautés se réapproprient le droit de résoudre leurs propres problèmes. La personne qui a commis l'offense, la ou les victime (s) (si quelqu'un a subi un tort direct) et la communauté doivent être consentants et travailler ensemble à la réintégration, grâce aux processus de responsabilisation, de restitution, de réparation, de conciliation et de guérison.

Prenant position pour le remplacement des prisons pour femmes par des alternatives non carcérales, les abolitionnistes citent : 1) la nature non menaçante de la majorité des actes illégaux commis par les femmes; 2) les enfants devenus orphelins et stigmatisés, ainsi que les problèmes d'éducation des enfants; 3) les conséquences des abus sexuels et physiques; 4) l'évidence que la moitié des meurtres commis par des femmes sont dirigés contre des agresseurs chroniques; 5) l'absence de logique en faveur de l'emprisonnement des femmes au-delà de la neutralisation des genres à l'ombre des institutions mâles (Immarigeon, 1987 : 3-4). Les femmes forment moins de $3 \%$ de la population dans les prisons fédérales canadiennes. Par conséquent, elles constituent une population toute désignée pour expérimenter des politiques de désinstitutionnalisation.

Se différenciant des opposants féministes à une justice punitive, de nombreuses autres militantes ont réclamé des sentences plus rapides, plus certaines et plus longues pour les hommes coupables de viol et d'agression sur des femmes et des enfants (Faith et Currie, 1993). Ces femmes résistent à une justice réparatrice parce qu'elles veulent que le coupable soit puni, elles veulent qu'il soit chassé de la communauté, car elles se sont rendu compte que les approches fondées sur la communauté prolongent souvent l'injustice. La justice est difficile à atteindre face à un déséquilibre structurel de pouvoir, tel qu'un mari abusif dans un cercle incluant sa femme abusée, ou un individu à faible revenu cherchant réparation d'un citoyen financièrement à l'aise, ou un enfant cherchant protection ou recours contre un adulte. S'ils ne tiennent pas compte de ce déséquilibre, les cercles de guérison ou de médiation peuvent avoir pour effet de faire taire encore plus la victime.

Le défi pour les militantes et les chercheures féministes qui oeuvrent en faveur de la désinstitutionnalisation, et dont la plupart sont abolitionnistes, consiste à trouver des façons de maintenir en dehors des prisons les délinquants qui ne posent aucune menace, tout en cherchant des moyens équitables d'affaiblir les idéologies pénales et les pratiques souvent violentes des traditions patriarcales. 
En Amérique du Nord, les féministes activistes, à l'intérieur comme à l'extérieur de la prison, continuent depuis trois décennies à promouvoir des idées de désinstitutionnalisation, en multipliant assemblées, forums, conférences et rassemblements pour réunir activistes populaires, les ex-prisonniers, les avocats, les chercheurs et les représentants des communautés les plus vulnérables à la criminalisation (Faith 2000). Par exemple, en 1973, à l'Institution pour femmes de la Californie, l'Organisation des détenues purgeant des peines de longue durée (Long-Termers Organization) a organisé une conférence sur les alternatives à la prison, à laquelle ont assisté la majorité des 600 détenues de l'époque (elles sont maintenant plus de 2000), et plus de 200 personnes de l'extérieur. Conscients de l'ironie de la situation, les participants ont échangé des idées sur l'abolition de la prison dans une prison à sécurité maximale, au cours d'une conférence animée par des femmes purgeant des sentences à vie.

En 1977, à Santa Cruz en Californie, de nombreuses ex-prisonnières ont assisté à une conférence sur les femmes en prison. C'était un précédent pour ces femmes que de pouvoir raconter en public ce qu'elles avaient vécu en prison, et de trouver une communauté prête à les appuyer. À Vancouver, en 1979, lors de la première conférence internationale sur «les femmes délinquantes», l'abolitionniste Claire Culhane $(1979 ; 1985)$ a mis sur pied un comité radical dont faisait partie la criminologue montréalaise Marie-Andrée Bertrand ainsi que d'autres universitaires, toutes protestant contre le maintien du statu quo pénal. En 1991, Madame Bertrand co-animait avec Kathleen Daly et Dorie Klein une conférence féministe internationale sur les femmes, la loi et le contrôle social.

À Phoenix en Arizona, en octobre 1993, lors d'assemblées de la American Society of Criminology (ASC), une réunion internationale mais à dominance américaine, la Division sur les femmes et le crime accepta de soutenir une résolution en faveur de la désinstitutionnalisation soumise par le groupe de travail de la division sur les femmes incarcérées, dans les termes suivants :

Il est, par la présente, décidé que les membres de l'ASC appuient les alternatives à l'incarcération pour les délinquants non violents. La surreprésentation des groupes historiquement privés de leurs droits de représentation dans les établissements carcéraux d'Amérique du Nord, l'évidence aiguë parmi les prisonniers d'expériences d'abus sexuel, physique et psychologique au cours de l'enfance, les coûts qu'impose à la société le fait de séparer les parents de leurs enfants à charge, et les pratiques de sentences discriminatoires, sont autant d'éléments pour indiquer un besoin de contester la per- 
pétuation de l'emprisonnement en réponse à des offenses commises par des délinquants jugés non violents. Nous recommandons fortement le développement et l'accroissement de services et de ressources communautaires dans le but explicite 1) de réduire le nombre de personnes condamnées à la prison; 2) de produire des modèles de désinstitutionnalisation qui vont promouvoir des principes d'équité sociale, 3) de fournir l'accès à des traitements appropriés (notre traduction).

Le ton «neutre quant au genre» de cette résolution était voulu. En Amérique du Nord, les injustices du système de la part des agences de justice criminelle sont, de façon constante et flagrante, commises contre les hommes d'origine africaine, hispanique ou autochtone. La Division sur les femmes et le crime souhaitait que le conseil d'administration de l'ASC endosse la résolution et présente le message anticarcéral aux législateurs nationaux. Sans grande surprise, la résolution fut rejetée. Cette demande de réduction du nombre de prisonniers, comme moyen de résistance au châtiment, vient défier un courant politique en faveur de l'augmentation des investissements dans les prisons. Les critiques n'arrivent pas à convaincre ceux qui ont le plus grand intérêt à soutenir le discours pénal.

En 1997, dans une maison de montagne au Québec lors d'une réunion maintenant connue comme le Rassemblement de Gatineau, une vingtaine de militantes canadiennes de la condition féminine et des droits de la personne se sont rencontrées pour élaborer des stratégies visant à trouver une solution aux abus commis sur les femmes dans les prisons. En 1998, à Berkeley en Californie, une conférence abolitionniste de la Résistance critique a attiré plus de 3500 personnes, dont beaucoup de femmes; Angela Davis était l'invitée de marque de cet événement. En 2000, la conférence internationale sur l'abolition de la peine (International Conference on Penal Abolition (ICOPA)) à Toronto a regroupé des centaines d'abolitionnistes convaincus, guidés par Ruth Morris et réclamant plus de fonds pour l'éducation, moins pour les prisons. En 2001, Kim Pate, directrice exécutive de l'Association canadienne des Sociétés Elizabeth Fry (ACSEF), et Lee Lakeman, de l'Association canadienne des centres contre le viol (ACCCV), ont tenu à Ottawa une conférence de Résistance des femmes pour mettre au point des stratégies de désinstitutionnalisation et de réduction des châtiments et de la violence faite aux femmes au Canada. Tous ces rassemblements ont une valeur éducative et visionnaire autant que stratégique : elles indiquent l'existence d'un mouvement populaire en faveur de changements transformationnels. 


\section{Conclusions}

Quand on fait un tour d'horizon sur l'évolution récente de la condition des femmes dans les prisons canadiennes, il apparaît clairement que, bien que certaines prisons soient beaucoup plus supportables que d'autres, aucune ne peut prétendre à l'avant-gardisme puisque la punition demeure leur raison d'être. Il en coûte près de 80000 \$ par année pour garder une femme en prison (CSC, 1997 : 12). Si cette femme était dangereuse, l'investissement serait justifiable; mais, la majorité des femmes emprisonnées ne constituent pas un danger pour autrui, et ces sommes seraient plus profitables appliquées à des ressources communautaires visant la réintégration et l'autosuffisance. Parmi toutes les prisons pour femmes au Canada, c'est la Okimaw Ohci Healing Loge qui se rapproche le plus d'une approche non pénale de l'incarcération, bien que la détention constitue une punition en soi. La question de savoir dans quelle mesure la loge peut maintenir une approche de guérison en ce qui a trait à l'incarcération en empiétant sur les méthodes «correctionnelles» reste à débattre.

Le Canada arrive en quatrième position au monde quant au taux d'incarcération, juste après les États-Unis, la Russie et l'Irlande du Nord, malgré l'évidence irréfutable que les prisons constituent un échec, quel que soit le point de vue adopté. Les politiciens privent d'argent le système d'éducation et d'autres programmes sociaux pour l'investir dans des institutions qui viennent aggraver ces mêmes problèmes qu'elles sont censées résoudre.

En 1998, Andy Scott, Solliciteur général en fonction, témoignait sa compréhension du dommage social qu'infligeaient les prisons en déclarant que la prison peut être «préjudiciable ou contre-productive» et que les contrevenants à la loi «pouvaient être traités de façon plus sûre et plus efficace par des programmes communautaires». Il a aussi exprimé le «besoin de mettre sur pied des alternatives efficaces à l'incarcération» (SolGen, 1998 : 4-6). Précédemment, il avait reconnu l'énorme gaspillage des fonds affectés à l'incarcération, admettant qu'il n'existait «aucun lien évident entre le crime dans la société et les niveaux d'incarcération». Plus encore, il mentionna une étude menée par le gouvernement démontrant que les programmes basés sur la communauté «semblaient comparables ou supérieurs aux mesures institutionnelles pour la réintégration en toute sécurité des délinquants» (SolGen, 1997 : 12; notre traduction).

Ole Ingstrup, alors commissaire du Service correctionnel du Canada, et responsable de l'exercice de l'autorité pour les 56 prisons et camps, 
s'accordait avec son patron pour déclarer que «des peines plus sévères n'entraînaient pas des communautés plus sûres» (Globe \& Mail, 1998). Dans le même courant, la Commission nationale des libérations conditionnelles déclare dans son manuel que «on obtient les plus faibles taux de récidive lorsque l'incarcération n'est utilisée qu'en dernier recours et qu'on offre du traitement dans des programmes à l'extérieur du système correctionnel» (CNLC, 1997 : 133 ; notre traduction). En effet, en 1996, l'adoption du projet de loi C-41 exigeait que les cours considèrent «toutes les alternatives à l'emprisonnement disponibles et qu'elles n'aient recours à l'emprisonnement que si aucun autre plan d'action ne pouvait garantir la sécurité de la société» (CCJA 1998 : 4 ; notre traduction). Le terme «justice réparatrice» a été officiellement adopté tant aux paliers provincial que fédéral, dérivant autant des traditions aborigènes que des innovations contemporaines dans une tentative pour mettre en place la détermination de la peine par la communauté, la médiation, le cercle de détermination de la peine, les cérémonies du cercle de guérison, ou d'autres moyens pour résoudre le crime à l'avantage des victimes et des communautés autant que des contrevenants à la loi (Landry, 1998).

Pour prévenir le crime, il faut se pencher sur les relations de pouvoir hiérarchique qui découlent de la criminalisation et de l'emprisonnement sélectifs et racistes. Si l'on arrivait à établir des communautés égalitaires avec une distribution plus équitable des ressources, on accomplirait un pas de géant vers la réduction du nombre de personnes incarcérées.

Les prisons sont dans la mire des féministes. En tant qu'institutions de l'État, les prisons sont de structure autant que d'idéologie patriarcales, une opération militariste et masculinisée tout à la fois inaptes à la socialisation de la plupart des femmes et à l'identification des rôles selon le genre. Les prisons ne guériront pas les femmes des abus et des souffrances physiques et émotionnelles subies durant des années, ou de la pauvreté qu'elles ont généralement endurée avant l'emprisonnement. Les prisons n'offrent pas aux femmes les ressources qui les aideraient à rebâtir leurs vies et à prendre soin de leurs enfants, ni à apprendre comment résister de façon constructive à la discrimination raciale. Il est grand temps de parler de démantèlement des prisons et de transférer les fonds publics dans l'éducation et les systèmes de soutien communautaire en faveur des mères célibataires sans emploi et de leurs enfants. Tel est le profil démographique féminin le plus vulnérable à l'emprisonnement. Entre-temps, nous pouvons œuvrer à édifier des communautés vouées à la justice sociale transformatrice. 


\section{Références}

Adler, F. (1983). Nations not obsessed with crime. Colorado : Fred B. Rothman and Company.

American Friends Service Committee (1971). Struggle for justice. New York : Hill and Wang.

Arbour, L. (1996). Commission of inquiry into certain events at the Prison for Femmes in Kingston. Ottawa : Solicitor General.

Balfour, G. (2000). Feminist therapy with Femmes in Prison: Working under the hegemony of correctionalism. In K. Hannah-Moffat \& M. Shaw (eds), An ideal prison? Critical essays on femmes's imprisonment in Canada (pp.94-102). Halifax : Fernwood.

Burkhart, K. W. (1973). Femmes in prison. Garden City : Doubleday \& Company, Inc.

Canadian Criminal Justice Association (1998). Prison overcrowding and the reintegration of offenders. Discussion Paper, March, Ottawa.

Carlen, P. (1990). Alternatives to femmes's imprisonment. Milton Keynes : Open University Press.

Chan, J.B.L., \& Ericson. R. (1981). Decarceration and the economy of penal reform. Toronto : University of Toronto, Centre of Criminology.

Christie, N. (1993). Crime control as industry. London : Routledge.

Service correctionnel du Canada (1997). Basic facts about corrections in Canada. Ottawa : Solicitor General.

Culhane, C. (1979). Barred from prison: A personal account. Vancouver : Pulp Press.

Culhane, C. (1985). Still barred from prison. Montreal : Black Rose Books.

de Haan, W. (1990). The politics of redress: Crime, punishment, and penal abolition. Boston : Unwin Hyman.

Dobash, R. P., Emerson Dobash, R., \& Gutteridge, S. (1986). The imprisonment of femmes. Oxford : Basil Blackwell Ltd.

Eaton, J. W. (1962). Stone walls not a prison make: The anatomy of planned administrative change. Springfield : Charles C. Thomas.

Faith, K. (1993). Unruly femmes: The politics of confinement and resistance. Vancouver : Press Gang Publishers.

Faith, K. (1995). Aboriginal femmes's Healing Lodge : Challenge to penal correctionalism? Journal of Human Justice, 6 (2),79-104.

Faith, K. (2000). Reflections on inside/out organizing. Social Justice, 27 (3), 158-67.

Faith, K., Gottfriedson, M., Joe, C., Leonard, W., \& McIvor, S. (1990). Native femmes in Canada: A quest for justice. Social Justice, 17 (3), 167-188.

Faith, K., \& Currie, D. (1993). Seeking shelter: A state of battered femmes. Vancouver : Press Gang Publishers.

Globe and Mail (1998). Incarceration rates too high, official says. March 17, p. B1.

Greenberg, D. F., \& Stender, F. (1972). The prison as a lawless agency. Buffalo Law Review, 799-839. 
Hannah-Moffat, K. (1991). Creating choices or repeating history?: Canadian female offenders and correctional reform. Social Justice, 13 (3), 184-203.

Hannah-Moffat, K. (2001). Punishment in disguise: Penal governance and federal imprisonment of femmes in Canada. Toronto : University of Toronto Press.

Hannah-Moffat, K., \& Shaw, M. (2000). An ideal prison? Critical essays on femmes's imprisonment in Canada. Halifax : Fernwood.

Horii, G. K. (2000). Processing humans. In K. Hannah-Moffat \& M. Shaw (eds), An ideal prison: Critical essays on femmes's imprisonment in Canada (pp.104116). Halifax : Fernwood.

Immarigeon, R. (1987). Femmes in prison. National Prison Project Journal, (Spring), ACLU, 2-5.

Kendall, K. (1994) Therapy behind prison walls: A contradiction in terms? Prison Service Journal, 96 (Nov.), 2-11.

Kendall, K. (2000). Psy-ence fiction: Governing female prisons through the psychological sciences. In K. Hannah-Moffat \& M. Shaw (eds), An ideal prison: Critical essays on femmes's imprisonment in Canada (pp.82-93). Halifax : Fernwood.

Knopp, F. et al. (1976). Instead of prisons. Syracuse : Prison Research Education Action Project.

Knopp, F. H. (1993). On radical feminism and abolition. In B.D. MacLean \& H.E. Pepinsky (eds), We who would take no prisoners (pp.53-61). Vancouver : Collective Press.

Landry, M. (1998). Réparatrice justice week: Community, victims, prisoners. Ottawa : CSC.

MacLean, B. D., \& Pepinsky, H.E. (1993). Preface to their edited We who would take no prisoners. Vancouver : Collective Press.

Mathiesen, T. (1986). The politics of abolition. Contemporary Crisis, 10(1), 81-94.

Mathiesen, T. (1990). Prison on trial : A critical assessment. London : Sage Publications.

Monture-Angus, P. (2000). Aboriginal femmes and correctional practice : Reflections on the Groupe de travail on Federally Sentenced Femmes. In K. Hannah-Moffat \& M. Shaw (eds), An ideal prison? Critical essays on femmes's imprisonment in Canada (pp.52-60). Halifax : Fernwood.

Morris, R. (1992). Réparatrice justice : Path to the future. Odyssey : Creative alternatives in criminal justice, (Spring), 90-95.

National Parole Board (1997). Risk assessment manual. Ottawa : Solicitor General.

Pate, K. (1997). Canadian Association of Elizabeth Fry Societies, annual report: Executive director. Ottawa.

Quinney, R., \& Pepinsky, H.E. (1990). The theory and practice of peacemaking in the development of radical criminology. Bloomington : Indiana University Press.

Shaw, M. (1993). Reforming federal femmes's imprisonment. In E. Adelberg \& C. Currie (eds), In conflict with the law: Femmes and the canadian justice system (pp.50-75). Vancouver : Press Gang Publishers.

Solicitor General's Special Committee on Provincially Incarcerated Femmes (1992). Blueprint for change. Province of Nova Scotia : Ministry of the Solicitor General. 
Solicitor General (1997). Service correctionnel du Canada : Performance Report. Ottawa, March 31.

Solicitor General (1998). Speaking notes for the Honourable Andy Scott, Solicitor General of Canada, to the Beyond Prison International Symposium. Kingston, March 17.

Sugar, F., \& Fox, L. (1989-90). Nistum Peyako Séht'wawin Iskwewak: Breaking Chains. Canadian Journal of Femmes and the Law/Revue juridique «la femme et le droit», 3 (2),465-482.

Task Force on Federally Sentenced Femmes (1990). Creating choices. Ottawa : Correctional Service Canada. 